\title{
3-uniform hypergraphs without a cycle of length five
}

\author{
Beka Ergemlidze \\ Department of Mathematics and Statistics \\ University of South Florida \\ Tampa, Florida, U.S.A.
}

beka.ergemlidze@gmail.com

\author{
Ervin Győri \\ Alfréd Rényi Institute of Mathematics \\ Budapest, Hungary \\ gyori.ervin@renyi.mta.hu
}

\author{
Abhishek Methuku \\ School of Mathematics \\ University of Birmingham \\ Birmingham, U.K.
}

abhishekmethuku@gmail.com

Submitted: Jun 19, 2019; Accepted: Apr 15, 2020; Published: May 1, 2020

(C) The authors. Released under the CC BY-ND license (International 4.0).

\begin{abstract}
In this paper we show that the maximum number of hyperedges in a 3-uniform hypergraph on $n$ vertices without a (Berge) cycle of length five is less than $(0.254+$ $o(1)) n^{3 / 2}$, improving an estimate of Bollobás and Györi.

We obtain this result by showing that not many 3-paths can start from certain subgraphs of the shadow.
\end{abstract}

Mathematics Subject Classifications: 05C65, 05D99

\section{Introduction}

A hypergraph $H=(V, E)$ is a family $E$ of distinct subsets of a finite set $V$. The members of $E$ are called hyperedges and the elements of $V$ are called vertices. A hypergraph is called 3-uniform if each member of $E$ has size 3. A hypergraph $H=(V, E)$ is called linear if every two hyperedges have at most one vertex in common.

A Berge cycle of length $k \geqslant 2$, denoted Berge- $C_{k}$, is an alternating sequence of distinct vertices and distinct edges of the form $v_{1}, h_{1}, v_{2}, h_{2}, \ldots, v_{k}, h_{k}$ where $v_{i}, v_{i+1} \in h_{i}$ for each $i \in\{1,2, \ldots, k-1\}$ and $v_{k}, v_{1} \in h_{k}$. (Note that if a hypergraph does not contain a Berge$C_{2}$, then it is linear.) This definition of a hypergraph cycle is the classical definition due to Berge. More generally, if $F=(V(F), E(F))$ is a graph and $\mathcal{Q}=(V(\mathcal{Q}), E(\mathcal{Q}))$ is a hypergraph, then we say $\mathcal{Q}$ is Berge-F if there is a bijection $\phi: E(F) \rightarrow E(\mathcal{Q})$ such that 
$e \subseteq \phi(e)$ for all $e \in E(F)$. In other words, given a graph $F$ we can obtain a Berge- $F$ by replacing each edge of $F$ with a hyperedge that contains it.

Given a family of graphs $\mathcal{F}$, we say that a hypergraph $\mathcal{H}$ is Berge- $\mathcal{F}$-free if for every $F \in \mathcal{F}$, the hypergraph $\mathcal{H}$ does not contain a Berge- $F$ as a subhypergraph. The maximum possible number of hyperedges in a Berge- $\mathcal{F}$-free 3 -uniform hypergraph on $n$ vertices is the Turán number of Berge- $\mathcal{F}$, and is denoted by $\operatorname{ex}_{3}(n, \mathcal{F})$. When $\mathcal{F}=\{F\}$ then we simply write $\operatorname{ex}_{3}(n, F)$ instead of $\operatorname{ex}_{3}(n,\{F\})$.

Determining $\operatorname{ex}_{3}\left(n,\left\{C_{2}, C_{3}\right\}\right)$ is basically equivalent to the famous $(6,3)$-problem. This was settled by Ruzsa and Szemerédi in their classical paper [23], showing that $n^{2-\frac{c}{\sqrt{\log n}}}<$ $\operatorname{ex}_{3}\left(n,\left\{C_{2}, C_{3}\right\}\right)=o\left(n^{2}\right)$ for some constant $c>0$. An important Turán-type extremal result for Berge cycles is due to Lazebnik and Verstraëte [21], who studied the maximum number of hyperedges in an $r$-uniform hypergraph containing no Berge cycle of length less than five (i.e., girth five). They showed the following.

Theorem 1 (Lazebnik, Verstraëte [21]).

$$
\operatorname{ex}_{3}\left(n,\left\{C_{2}, C_{3}, C_{4}\right\}\right)=\frac{1}{6} n^{3 / 2}+o\left(n^{3 / 2}\right) .
$$

The systematic study of the Turán number of Berge cycles started with the study of Berge triangles by Györi [15], and continued with the study of Berge five cycles by Bollobás and Győri [1] who showed the following.

Theorem 2 (Bollobás, Győri [1]).

$$
(1+o(1)) \frac{n^{3 / 2}}{3 \sqrt{3}} \leqslant \operatorname{ex}_{3}\left(n, C_{5}\right) \leqslant \sqrt{2} n^{3 / 2}+4.5 n .
$$

The following example of Bollobás and Györi proves the lower bound in Theorem 2.

Bollobás-Györi Example. Take a $C_{4}$-free bipartite graph $G_{0}$ with $n / 3$ vertices in each part and $(1+o(1))(n / 3)^{3 / 2}$ edges. In one part, replace each vertex $u$ of $G_{0}$ by a pair of two new vertices $u_{1}$ and $u_{2}$, and add the triple $u_{1} u_{2} v$ for each edge $u v$ of $G_{0}$. It is easy to check that the resulting hypergraph $H$ does not contain a Berge cycle of length 5 . Moreover, the number of hyperedges in $H$ is the same as the number of edges in $G_{0}$.

In this paper, we improve Theorem 2 as follows.

\section{Theorem 3.}

$$
\operatorname{ex}_{3}\left(n, C_{5}\right)<(1+o(1)) 0.254 n^{3 / 2}
$$

Roughly speaking, our main idea in proving the above theorem is to analyze the structure of a Berge- $C_{5}$-free hypergraph, and use this structure to efficiently bound the number of paths of length 3 that start from certain dense subgraphs (e.g., triangle, $K_{4}$ ) of the 2-shadow. This bound is then combined with the lower bound on the number of paths of length 3 provided by the Blakley-Roy inequality [2]. We prove Theorem 3 in Section 2. 
Ergemlidze, Győri and Methuku [3] considered the analogous question for linear hypergraphs and proved that $\operatorname{ex}_{3}\left(n,\left\{C_{2}, C_{5}\right\}\right)=n^{3 / 2} / 3 \sqrt{3}+o\left(n^{3 / 2}\right)$. Surprisingly, even though their lower bound is the same as the lower bound in Theorem 2, the linear hypergraph that they constructed in [3] is very different from the hypergraph used in the BollobásGyöri example discussed above - the latter is far from being linear. In [3], the authors also strengthened Theorem 1 by showing that $\operatorname{ex}_{3}\left(n,\left\{C_{2}, C_{3}, C_{4}\right\}\right) \sim \operatorname{ex}_{3}\left(n,\left\{C_{2}, C_{4}\right\}\right)$. Recently, $\operatorname{ex}_{3}\left(n, C_{4}\right)$ was studied in [5]. See [6] for results on the maximum number of hyperedges in an $r$-uniform hypergraph of girth six.

Győri and Lemons $[16,17]$ generalized Theorem 2 to Berge cycles of any given length and proved bounds on $\operatorname{ex}_{r}\left(n, C_{2 k+1}\right)$ and $\operatorname{ex}_{r}\left(n, C_{2 k}\right)$. These bounds were improved by Füredi and Özkahya [9], Jiang and Ma [19], Gerbner, Methuku and Vizer [11]. Recently Füredi, Kostochka and Luo [7] started the study of the maximum size of an $n$-vertex $r$-uniform hypergraph without any Berge cycle of length at least $k$. This study has been continued in $[8,18,20,4]$.

General results for Berge- $F$-free hypergraphs have been obtained in $[12,13,10]$ and the Turán numbers of Berge- $K_{2, t}$ and Berge cliques, among others, were studied in [24, $22,11,14,10]$.

\section{Notation}

We introduce some important notations and definitions used throughout the paper.

- Length of a path is the number of edges in the path. We usually denote a path $v_{0}, v_{1}, \ldots, v_{k}$, simply as $v_{0} v_{1} \ldots v_{k}$.

- For convenience, an edge $\{a, b\}$ of a graph or a pair of vertices $a, b$ is referred to as $a b$. A hyperedge $\{a, b, c\}$ is written simply as $a b c$.

- For a hypergraph $H$ (or a graph $G$ ), for convenience, we sometimes use $H$ (or $G$ ) to denote the edge set of the hypergraph $H$ (or $G$ respectively). Thus the number of edges in $H$ is $|H|$.

- Given a graph $G$ and a subset of its vertices $S$, let the subgraph of $G$ induced by $S$ be denoted by $G[S]$.

- For a hypergraph $H$, let $\partial H=\{a b \mid a b \subset e \in E(H)\}$ denote its 2-shadow graph.

- For a hypergraph $H$, the neighborhood of $v$ in $H$ is defined as

$$
N(v)=\{x \in V(H) \backslash\{v\} \mid v, x \in h \text { for some } h \in E(H)\} .
$$

- For a hypergraph $H$ and a pair of vertices $u, v \in V(H)$, let $\operatorname{codeg}(v, u)$ denote the number of hyperedges of $H$ containing the pair $\{u, v\}$.

\section{Proof of Theorem 3}

Let $H$ be a hypergraph on $n$ vertices without a Berge 5 -cycle and let $G=\partial H$ be the 2 -shadow of $H$. First we introduce some definitions. 
Definition 4. A pair $x y \in \partial H$ is called thin if $\operatorname{codeg}(x y)=1$, otherwise it is called fat. We say a hyperedge $a b c \in H$ is thin if at least two of the pairs $a b, b c, a c$ are thin.

Definition 5. We say a set of hyperedges (or a hypergraph) is tightly-connected if it can be obtained by starting with a hyperedge and adding hyperedges one by one, such that every added hyperedge intersects with one of the previous hyperedges in 2 vertices.

Definition 6. A block in $H$ is a maximal set of tightly-connected hyperedges.

Definition 7. For a block $B$, a maximal subhypergraph of $B$ without containing thin hyperedges is called the core of the block.

Let $K_{4}^{3}$ denote the complete 3 -uniform hypergraph on 4 vertices. A crown of size $k$ is a set of $k \geqslant 1$ hyperedges of the form $a b c_{1}, a b c_{2}, \ldots, a b c_{k}$. Below we define 2 specific hypergraphs:

- Let $F_{1}$ be a hypergraph consisting of exactly 3 hyperedges on 4 vertices (i.e., $K_{4}^{3}$ minus an edge).

- For distinct vertices $a, b, c, d$ and $o$, let $F_{2}$ be the hypergraph consisting of hyperedges $o a b, o b c$, ocd and oda.

Lemma 8. Let $B$ be a block of $H$, and let $\mathcal{B}$ be a core of $B$. Then $\mathcal{B}$ is either $\emptyset, K_{4}^{3}, F_{1}, F_{2}$ or a crown of size $k$ for some $k \geqslant 1$.

Proof. If $\mathcal{B}=\emptyset$, we are done, so let us assume $\mathcal{B} \neq \emptyset$. Since $B$ is tightly-connected and it can be obtained by adding thin hyperedges to $\mathcal{B}$, it is easy to see that $\mathcal{B}$ is also tightly-connected. Thus if $\mathcal{B}$ has at most two hyperedges, then it is a crown of size 1 or 2 and we are done. Therefore, in the rest of the proof we will assume that $\mathcal{B}$ contains at least 3 hyperedges.

If $\mathcal{B}$ contains at most 4 vertices then it is easy to see that $\mathcal{B}$ is either $K_{4}^{3}$ or $F_{1}$. So assume that $\mathcal{B}$ has at least 5 vertices (and at least 3 hyperedges). Since $\mathcal{B}$ is not a crown, there exists a tight path of length 3 , say $a b c, b c d, c d e$. Since $a b c$ is in the core, one of the pairs $a b$ or $a c$ is fat, so there exists a hyperedge $h \neq a b c$ containing either $a b$ or $a c$. Similarly there exists a hyperedge $f \neq c d e$ and $f$ contains $e d$ or $e c$. If $h=f$ then $\mathcal{B} \supseteq F_{2}$. However, it is easy to see that $F_{2}$ cannot be extended to a larger tightly-connected set of hyperedges without creating a Berge 5 -cycle, so in this case $\mathcal{B}=F_{2}$. If $h \neq f$ then the hyperedges $h, a b c, b c d, c d e, f$ create a Berge 5 -cycle in $H$, a contradiction. This completes the proof of the lemma.

Observation 9. Let $B$ be a block of $H$ and let $\mathcal{B}$ be the core of $B$. If $\mathcal{B}=\emptyset$ then the block $B$ is a crown, and if $\mathcal{B} \neq \emptyset$ then every fat pair of $B$ is contained in $\partial \mathcal{B}$.

Edge Decomposition of $\boldsymbol{G}=\boldsymbol{\partial H}$. We define a decomposition $\mathcal{D}$ of the edges of $G$ into paths of length 2 , triangles and $K_{4}$ 's such as follows:

Let $B$ be a block of $H$ and $\mathcal{B}$ be its core. 
If $\mathcal{B}=\emptyset$, then $B$ is a crown-block $\left\{a b c_{1}, a b c_{2}, \ldots, a b c_{k}\right\}$ (for some $k \geqslant 1$ ); we partition $\partial B$ into the triangle $a b c_{1}$ and paths $a c_{i} b$ where $2 \leqslant i \leqslant k$.

If $\mathcal{B} \neq \emptyset$, then our plan is to first partition $\partial B \backslash \partial \mathcal{B}$. If $a b c \in B \backslash \mathcal{B}$, then $a b c$ is a thin hyperedge, so it contains at least 2 thin pairs, say $a b$ and $b c$. We claim that the pair $a c$ is in $\partial \mathcal{B}$. Indeed, $a c$ has to be a fat pair, otherwise the block $B$ consists of only one hyperedge $a b c$, so $\mathcal{B}=\emptyset$ contradicting the assumption. So by Observation $9, a c$ has to be a pair in $\partial \mathcal{B}$. For every $a b c \in B \backslash \mathcal{B}$ such that $a b$ and $b c$ are thin pairs, add the 2-path $a b c$ to the edge decomposition $\mathcal{D}$. This partitions all the edges in $\partial B \backslash \partial \mathcal{B}$ into paths of length 2 . So all we have left is to partition the edges of $\partial \mathcal{B}$.

- If $\mathcal{B}$ is a crown $\left\{a b c_{1}, a b c_{2}, \ldots, a b c_{k}\right\}$ for some $k \geqslant 1$, then we partition $\partial B$ into the triangle $a b c_{1}$ and paths $a c_{i} b$ where $2 \leqslant i \leqslant k$.

- If $\mathcal{B}=F_{1}=\{a b c, b c d, a c d\}$ then we partition $\partial \mathcal{B}$ into 2-paths $a b c, b d c$ and $c a d$.

- If $\mathcal{B}=F_{2}=\{o a b, o b c, o c d, o d a\}$ then we partition $\partial \mathcal{B}$ into 2-paths abo, bco, cdo and dao.

- Finally, if $\mathcal{B}=K_{4}^{3}=\{a b c, a b d, a c d, b c d\}$ then we partition $\partial \mathcal{B}$ as $K_{4}$, i.e., we add $\partial \mathcal{B}=K_{4}$ as an element of $\mathcal{D}$.

Clearly, by Lemma 8 we have no other cases left. Thus all of the edges of the graph $G$ are partitioned into paths of length 2 , triangles and $K_{4}$ 's.

\section{Observation 10.}

(a) If $D$ is a triangle that belongs to $\mathcal{D}$, then there is a hyperedge $h \in H$ such that $D=\partial h$.

(b) If abc is a 2-path that belongs to $\mathcal{D}$, then abc $\in H$. Moreover ac is a fat pair.

(c) If $D$ is a $K_{4}$ that belongs to $\mathcal{D}$, then there exists $F=K_{4}^{3} \subseteq H$ such that $D=\partial F$.

Let $\alpha_{1}|G|$ and $\alpha_{2}|G|$ be the number of edges of $G$ that are contained in triangles and 2-paths of the edge-decomposition $\mathcal{D}$ of $G$, respectively. So $\left(1-\alpha_{1}-\alpha_{2}\right)|G|$ edges of $G$ belong to the $K_{4}$ 's in $\mathcal{D}$.

Claim 11. We have,

$$
|H|=\left(\frac{\alpha_{1}}{3}+\frac{\alpha_{2}}{2}+\frac{2\left(1-\alpha_{1}-\alpha_{2}\right)}{3}\right)|G|
$$

Proof. Let $B$ be a block with the core $\mathcal{B}$. Recall that for each hyperedge $h \in B \backslash \mathcal{B}$, we have added exactly one 2 -path or a triangle to $\mathcal{D}$.

Moreover, because of the way we partitioned $\partial \mathcal{B}$, it is easy to check that in all of the cases except when $\mathcal{B}=K_{4}^{3}$, the number of hyperedges of $\mathcal{B}$ is the same as the number of elements of $\mathcal{D}$ that $\partial \mathcal{B}$ is partitioned into; these elements being 2-paths and triangles. On the other hand, if $\mathcal{B}=K_{4}^{3}$, then the number of hyperedges of $\mathcal{B}$ is 4 but we added only one element to $\mathcal{D}$ (namely $K_{4}$ ). 
This shows that the number of hyperedges of $H$ is equal to the number of elements of $\mathcal{D}$ that are 2-paths or triangles plus the number of hyperedges which are in copies of $K_{4}^{3}$ in $H$, i.e., 4 times the number of $K_{4}$ 's in $\mathcal{D}$. Since $\alpha_{1}|G|$ edges of $G$ are in 2-paths, the number of elements of $\mathcal{D}$ that are 2-paths is $\alpha_{1}|G| / 2$. Similarly, the number of elements of $\mathcal{D}$ that are triangles is $\alpha_{2}|G| / 3$, and the number of $K_{4}$ 's in $\mathcal{D}$ is $\left(1-\alpha_{1}-\alpha_{2}\right)|G| / 6$. Combining this with the discussion above finishes the proof of the claim.

The link of a vertex $v$ is the graph consisting of the edges $\{u w \mid u v w \in H\}$ and is denoted by $L_{v}$.

Claim 12. $\left|L_{v}\right| \leqslant 2|N(v)|$.

Proof. First let us notice that there is no path of length 5 in $L_{v}$. Indeed, otherwise, there exist vertices $v_{0}, v_{1}, \ldots, v_{5}$ such that $v v_{i-1} v_{i} \in H$ for each $1 \leqslant i \leqslant 5$ which means there is a Berge 5 -cycle in $H$ formed by the hyperedges containing the pairs $v v_{1}, v_{1} v_{2}, v_{2} v_{3}, v_{3} v_{4}, v_{4} v$, a contradiction. So by the Erdös-Gallai theorem $\left|L_{v}\right| \leqslant \frac{5-1}{2}|N(v)|$, proving the claim.

Lemma 13. Let $v \in V(H)$ be an arbitrary vertex, then the number of edges in $G[N(v)]$ is less than $8|N(v)|$.

Proof. Let $G_{v}$ be a subgraph of $G$ on a vertex set $N(v)$, such that $x y \in G_{v}$ if and only if there exists a vertex $z \neq v$ such that $x y z \in H$. Then each edge of $G[N(v)]$ belongs to either $L_{v}$ or $G_{v}$, so $|G[N(v)]| \leqslant\left|L_{v}\right|+\left|G_{v}\right|$. Combining this with Claim 12, we get $|G[N(v)]| \leqslant\left|G_{v}\right|+2|N(v)|$. So it suffices to prove that $\left|G_{v}\right|<6|N(v)|$.

First we will prove that there is no path of length 12 in $G_{v}$. Let us assume by contradiction that $P=v_{0}, v_{1}, \ldots, v_{12}$ is a path in $G_{v}$. Since for each pair of vertices $v_{i}, v_{i+1}$, there is a hyperedge $v_{i} v_{i+1} x$ in $H$ where $x \neq v$, we can conclude that there is a subsequence $u_{0}, u_{1}, \ldots, u_{6}$ of $v_{0}, v_{1}, \ldots, v_{12}$ and a sequence of distinct hyperedges $h_{1}, h_{2}, \ldots, h_{6}$, such that $u_{i-1} u_{i} \subset h_{i}$ and $v \notin h_{i}$ for each $1 \leqslant i \leqslant 6$. Since $u_{0}, u_{3}, u_{6} \in N(v)$ there exist hyperedges $f_{1}, f_{2}, f_{3} \in H$ such that $v u_{0} \subset f_{1}, v u_{3} \subset f_{2}$ and $v u_{6} \subset f_{3}$. Clearly, either $f_{1} \neq f_{2}$ or $f_{2} \neq f_{3}$. In the first case the hyperedges $f_{1}, h_{1}, h_{2}, h_{3}, f_{2}$, and in the second case the hyperedges $f_{2}, h_{4}, h_{5}, h_{6}, f_{3}$ form a Berge 5 -cycle in $H$, a contradiction.

Therefore, there is no path of length 12 in $G_{v}$, so by the Erdös-Gallai theorem, the number of edges in $G_{v}$ is at most $\frac{12-1}{2}|N(v)|<6|N(v)|$, as required.

\subsection{Relating the hypergraph degree to the degree in the shadow}

For a vertex $v \in V(H)=V(G)$, let $d(v)$ denote the degree of $v$ in $H$ and let $d_{G}(v)$ denote the degree of $v$ in $G$ (i.e., $d_{G}(v)$ is the degree in the shadow).

Clearly $d_{G}(v) \leqslant 2 d(v)$. Moreover, $d(v)=\left|L_{v}\right|$ and $d_{G}(v)=|N(v)|$. So by Claim 12 , we have

$$
\frac{d_{G}(v)}{2} \leqslant d(v) \leqslant 2 d_{G}(v)
$$

Let $\bar{d}$ and $\bar{d}_{G}$ be the average degrees of $H$ and $G$ respectively.

Suppose there is a vertex $v$ of $H$, such that $d(v)<\bar{d} / 3$. Then we may delete $v$ and all the edges incident to $v$ from $H$ to obtain a graph $H^{\prime}$ whose average degree is more than 
$3(n \bar{d} / 3-\bar{d} / 3) /(n-1)=\bar{d}$. Then it is easy to see that if the theorem holds for $H^{\prime}$, then it holds for $H$ as well. Repeating this procedure, we may assume that for every vertex $v$ of $H, d(v) \geqslant \bar{d} / 3$. Therefore, by (1), we may assume that the degree of every vertex of $G$ is at least $\bar{d} / 6$.

\subsection{Counting paths of length 3}

Definition 14. A 2-path in $\partial H$ is called bad if both of its edges are contained in a triangle of $\partial H$, otherwise it is called good.

Lemma 15. For any vertex $v \in V(G)$ and a set $M \subseteq N(v)$, let $\mathcal{P}$ be the set of the good 2-paths vxy such that $x \in M$. Let $M^{\prime}=\{y \mid$ vxy $\in \mathcal{P}\}$ then $|\mathcal{P}|<2\left|M^{\prime}\right|+48 d_{G}(v)$.

Proof. Let $B_{\mathcal{P}}=\left\{x y \mid x \in M, y \in M^{\prime}, x y \in G\right\}$ be a bipartite graph, clearly $\left|B_{\mathcal{P}}\right|=|\mathcal{P}|$. Let $E=\{x y z \in H \mid x, y \in N(v), \operatorname{codeg}(x, y) \leqslant 2\}$. By Lemma $13,|E| \leqslant 2 \cdot 8|N(v)|$ so the number of edges of 2-shadow of $E$ is $|\partial E| \leqslant 48|N(v)|$. Let $B=\left\{x y \in B_{\mathcal{P}} \mid \exists z \in\right.$ $V(H), x y z \in H \backslash E\}$. Then clearly,

$$
|B| \geqslant\left|B_{\mathcal{P}}\right|-|\partial E| \geqslant|\mathcal{P}|-48|N(v)|=|\mathcal{P}|-48 d_{G}(v) .
$$

Let $d_{B}(x)$ denote the degree of a vertex $x$ in the graph $B$.

Claim 16. For every $y \in M^{\prime}$ such that $d_{B}(y)=k \geqslant 3$, there exists a set of $k-2$ vertices $S_{y} \subseteq M^{\prime}$ such that $\forall w \in S_{y}$ we have $d_{B}(w)=1$. Moreover, $S_{y} \cap S_{z}=\emptyset$ for any $y \neq z \in M^{\prime}$ (with $d_{B}(y), d_{B}(z) \geqslant 3$ ).

Proof. Let $y x_{1}, y x_{2}, \ldots, y x_{k} \in B$ be the edges of $B$ incident to $y$. For each $1 \leqslant j \leqslant k$ let $f_{j} \in H$ be a hyperedge such that $v x_{j} \subset f_{j}$. For each $y x_{i} \in B$ clearly there is a hyperedge $y x_{i} w_{i} \in H \backslash E$.

We claim that for each $1 \leqslant i \leqslant k, w_{i} \in M^{\prime}$. It is easy to see that $w_{i} \in N(v)$ or $w_{i} \in M^{\prime}$ (because $v x_{i} w_{i}$ is a 2-path in $G$ ). Assume for a contradiction that $w_{i} \in N(v)$, then since $y x_{i} w_{i} \notin E$ we have, $\operatorname{codeg}\left(x_{i}, w_{i}\right) \geqslant 3$. Let $f \in H$ be a hyperedge such that $v w_{i} \subset f$. Now take $j \neq i$ such that $x_{j} \neq w_{i}$. If $f_{j} \neq f$ then since $\operatorname{codeg}\left(x_{i}, w_{i}\right) \geqslant 3$ there exists a hyperedge $h \supset x_{i} w_{i}$ such that $h \neq f$ and $h \neq x_{i} w_{i} y$, then the hyperedges $f, h, x_{i} w_{i} y, y x_{j} w_{j}, f_{j}$ form a Berge 5 -cycle. So $f_{j}=f$, therefore $f_{j} \neq f_{i}$. Similarly in this case, there exists a hyperedge $h \supset x_{i} w_{i}$ such that $h \neq f_{i}$ and $h \neq x_{i} w_{i} y$, therefore the hyperedges $f_{i}, h, x_{i} w_{i} y, y x_{j} w_{j}, f_{j}$ form a Berge 5 -cycle, a contradiction. So we proved that $w_{i} \in M^{\prime}$ for each $1 \leqslant i \leqslant k$.

Claim 17. For all but at most 2 of the $w_{i}$ 's (where $1 \leqslant i \leqslant k$ ), we have $d_{B}\left(w_{i}\right)=1$.

Proof. If $d_{B}\left(w_{i}\right)=1$ for all $1 \leqslant i \leqslant k$ then we are done, so we may assume that there is $1 \leqslant i \leqslant k$ such that $d_{B}\left(w_{i}\right) \neq 1$.

For each $1 \leqslant i \leqslant k, w_{i} \in M^{\prime}$ and $x_{i} w_{i} \in \partial(H \backslash E)$ (because $x_{i} w_{i} y \in H \backslash E$ ), so it is clear that $d_{B}\left(w_{i}\right) \geqslant 1$. So $d_{B}\left(w_{i}\right)>1$. Then there is a vertex $x \in M \backslash\left\{x_{i}\right\}$ such that $w_{i} x \in B$. Let $f, h \in H$ be hyperedges with $w_{i} x \in h$ and $x v \in f$. If there are $j, l \in\{1,2, \ldots, k\} \backslash\{i\}$ such that $x, x_{j}$ and $x_{l}$ are all different from each other, then 
clearly, either $f \neq f_{j}$ or $f \neq f_{l}$, so without loss of generality we may assume $f \neq f_{j}$. Then the hyperedges $f, h, w_{i} x_{i} y, y w_{j} x_{j}, f_{j}$ create a Berge cycle of length 5 , a contradiction. So there are no $j, l \in\{1,2, \ldots, k\} \backslash\{i\}$ such that $x, x_{j}$ and $x_{l}$ are all different from each other. Clearly this is only possible when $k<4$ and there is a $j \in\{1,2,3\} \backslash\{i\}$ such that $x=x_{j}$. Let $l \in\{1,2,3\} \backslash\{i, j\}$. If $f_{j} \neq f_{l}$ then the hyperedges $f_{j}, h, w_{i} x_{i} y, y w_{l} x_{l}, f_{l}$ form a Berge 5-cycle. Therefore $f_{j}=f_{l}$. So we proved that $d_{B}\left(w_{i}\right) \neq 1$ implies that $k=3$ and for $\{j, l\}=\{1,2,3\} \backslash\{i\}$, we have $f_{j}=f_{l}$. So if $d_{B}\left(w_{i}\right) \neq 1$ and $d_{B}\left(w_{j}\right) \neq 1$ we have $f_{j}=f_{l}$ and $f_{i}=f_{l}$, which is impossible. So $d_{B}\left(w_{j}\right)=1$. So we proved that if for any $1 \leqslant i \leqslant k, d_{B}\left(w_{i}\right) \neq 1$ then $k=3$ and all but at most 2 of the vertices in $\left\{w_{1}, w_{2}, w_{3}\right\}$ have degree 1 in the graph $B$, as desired.

We claim that for any $i \neq j$ where $d_{B}\left(w_{i}\right)=d_{B}\left(w_{j}\right)=1$ we have $w_{i} \neq w_{j}$. Indeed, if there exists $i \neq j$ such that $w_{i}=w_{j}$ then $w_{i} x_{j}$ and $w_{i} x_{i}$ are both adjacent to $w_{i}$ in the graph $B$ which contradicts to $d_{B}\left(w_{i}\right)=1$. So using the above claim, we conclude that the set $\left\{w_{1}, w_{2}, \ldots, w_{k}\right\}$ contains at least $k-2$ distinct elements with each having degree one in the graph $B$, so we can set $S_{y}$ to be the set of these $k-2$ elements. (Then of course $\forall w_{i} \in S_{y}$ we have $d_{B}\left(w_{i}\right)=1$.)

Now we have to prove that for each $z \neq y$ we have $S_{y} \cap S_{z}=\emptyset$. Assume by contradiction that $w_{i} \in S_{z} \cap S_{y}$ for some $z \neq y$. That is, there is some hyperedge $u w_{i} z \in H \backslash E$ where $u \in M$, moreover $u=x_{i}$ otherwise $d_{B}\left(w_{i}\right)>1$. So we have a hyperedge $x_{i} w_{i} z \in H \backslash E$ for some $z \in M^{\prime} \backslash\{y\}$. Let $j, l \in\{1,2, \ldots, k\} \backslash\{i\}$ such that $j \neq l$. Recall that $x_{j} v \subset f_{j}$ and $x_{l} v \subset f_{l}$. Clearly either $f_{j} \neq f_{i}$ or $f_{l} \neq f_{i}$ so without loss of generality we can assume $f_{j} \neq f_{i}$. Then it is easy to see that the hyperedges $f_{j}, x_{j} w_{j} y, y x_{i} w_{i}, w_{i} z x_{i}, f_{i}$ are all different and they create a Berge 5 -cycle $\left(x_{j} w_{j} y \neq y x_{i} w_{i}\right.$ because $\left.x_{j} \neq w_{i}\right)$.

For each $x \in M^{\prime}$ with $d_{B}(x)=k \geqslant 3$, let $S_{x}$ be defined as in Claim 16. Then the average of the degrees of the vertices in $S_{x} \cup\{x\}$ in $B$ is $\left(k+\left|S_{x}\right|\right) /(k-1)=(2 k-2)(k-1)=$ 2. Since the sets $S_{x} \cup x$ (with $x \in M^{\prime}, d_{B}(x) \geqslant 3$ ) are disjoint, we can conclude that average degree of the set $M^{\prime}$ is at most 2. Therefore $2\left|M^{\prime}\right| \geqslant|B|$. So by (2) we have $2\left|M^{\prime}\right| \geqslant|B|>|\mathcal{P}|-48 d_{G}(V)$, which completes the proof of the lemma.

Claim 18. We may assume that the maximum degree in the graph $G$ is less than $160 \sqrt{n}$ when $n$ is large enough.

Proof. Let $v$ be an arbitrary vertex with $d_{G}(v)=C \bar{d}$ for some constant $C>0$. Let $\mathcal{P}$ be the set of the good 2-paths starting from the vertex $v$. Then applying Lemma 15 with $M=N(v)$ and $M^{\prime}=\{y \mid v x y \in \mathcal{P}\}$, we have $|\mathcal{P}|<2\left|M^{\prime}\right|+48 d_{G}(v)<2 n+48 \cdot C \bar{d}$. Since the minimum degree in $G$ is at least $\bar{d} / 6$, the number of (ordered) 2-paths starting from $v$ is at least $d(v) \cdot(\bar{d} / 6-1)=C \bar{d} \cdot(\bar{d} / 6-1)$. Notice that the number of (ordered) bad 2-paths starting at $v$ is the number of 2-paths $v x y$ such that $x, y \in N(v)$. So by Lemma 13, this is at most $2 \cdot 8|N(v)|=16 C \bar{d}$, so the number of good 2-paths is at least $C \bar{d} \cdot(\bar{d} / 6-17)$. So $|\mathcal{P}| \geqslant C \bar{d} \cdot(\bar{d} / 6-17)$. Thus we have

$$
C \bar{d} \cdot(\bar{d} / 6-17) \leqslant|\mathcal{P}|<2 n+48 C \bar{d} .
$$


So $C \bar{d}(\bar{d} / 6-65)<2 n$. Therefore, $6 C(\bar{d} / 6-65)^{2}<2 n$, i.e., $\bar{d}<6 \sqrt{n / 3 C}+390$, so $|H|=n \bar{d} / 3 \leqslant 2 n \sqrt{n / 3 C}+130 n$. If $C \geqslant 36$ we get that $|H| \leqslant \frac{n^{3 / 2}}{3 \sqrt{3}}+130 n=\frac{n^{3 / 2}}{3 \sqrt{3}}+O(n)$, proving Theorem 3. So we may assume $C<36$.

Theorem 2 implies that

$$
|H|=n \bar{d} / 3 \leqslant \sqrt{2} n^{3 / 2}+4.5 n
$$

so $\bar{d} \leqslant 3 \sqrt{2} \sqrt{n}+13.5$. So combining this with the fact that $C<36$, we have $d_{G}(v)=$ $C \bar{d}<108 \sqrt{2} \sqrt{n}+486<160 \sqrt{n}$ for large enough $n$.

Combining Lemma 15 and Claim 18, we obtain the following.

Lemma 19. For any vertex $v \in V(G)$ and a set $M \subseteq N(v)$, let $\mathcal{P}$ be the set of good 2-paths vxy such that $x \in M$. Let $M^{\prime}=\{y \mid$ vxy $\in \mathcal{P}\}$ then $|\mathcal{P}|<2\left|M^{\prime}\right|+7680 \sqrt{n}$ when $n$ is large enough.

Definition 20. A 3-path $x_{0}, x_{1}, x_{2}, x_{3}$ is called good if both 2-paths $x_{0}, x_{1}, x_{2}$ and $x_{1}, x_{2}, x_{3}$ are good 2-paths.

Claim 21. The number of (ordered) good 3-paths in $G$ is at least $n \bar{d}_{G}^{3}-C_{0} n^{3 / 2} \bar{d}_{G}$ for some constant $C_{0}>0$ (for large enough $n$ ).

Proof. First we will prove that the number of (ordered) 3-walks that are not good 3-paths is at most $5440 n^{3 / 2} \bar{d}_{G}$.

For any vertex $x \in V(H)$ if a path $y x z$ is a bad 2-path then $z y$ is an edge of $G$, so the number of (ordered) bad 2-paths whose middle vertex is $x$, is at most 2 times the number of edges in $G[N(x)]$, which is less than $2 \cdot 8|N(x)|=16 d_{G}(x)$ by Lemma 13 . The number of 2 -walks which are not 2-paths and whose middle vertex is $x$ is exactly $d_{G}(x)$. So the total number of (ordered) 2-walks that are not good 2-paths is at most $\sum_{x \in V(H)} 17 d_{G}(x)=17 n \bar{d}_{G}$.

Notice that, by definition, any (ordered) 3-walk that is not a good 3-path must contain a 2-walk that is not a good 2-path. Moreover, if $x y z$ is a 2-walk that is not a good 2-path, then the number of 3 -walks in $G$ containing it is at most $d_{G}(x)+d_{G}(z)<320 \sqrt{n}$ (for large enough $n$ ) by Claim 18. Therefore, the total number of (ordered) 3-walks that are not good 3-paths is at most $17 n \bar{d}_{G} \cdot 320 \sqrt{n}=5440 n^{3 / 2} \bar{d}_{G}$.

By the Blakley-Roy inequality, the total number of (ordered) 3-walks in $G$ is at least $n \bar{d}_{G}^{3}$. By the above discussion, all but at most $5440 n^{3 / 2} \bar{d}_{G}$ of them are good 3-paths, so letting $C_{0}=5440$ completes the proof of the claim.

Claim 22. Let $\{a, b, c\}$ be the vertex set of a triangle that belongs to $\mathcal{D}$. (By Observation 10 (a) $a b c \in H$.) Then the number of good 3-paths whose first edge is ab, bc or ca is at most $8 n+C_{1} \sqrt{n}$ for some constant $C_{1}$ and for large enough $n$.

Proof. For each $\{x, y\} \subset\{a, b, c\}$, let $S_{x y}=N(x) \cap N(y) \backslash\{a, b, c\}$. For each $x \in\{a, b, c\}$, let $S_{x}=N(x) \backslash(N(y) \cup N(z) \cup\{a, b, c\})$ where $\{y, z\}=\{a, b, c\} \backslash\{x\}$. 
For each $x \in\{a, b, c\}$, let $\mathcal{P}_{x}$ be the set of good 2-paths $x u v$ where $u \in S_{x}$. Let $S_{x}^{\prime}=\left\{v \mid x u v \in \mathcal{P}_{x}\right\}$. For each $\{x, y\} \subset\{a, b, c\}$, let $\mathcal{P}_{x y}$ be the set of good 2-paths $x u v$ and yuv where $u \in S_{x y}$. Let $S_{x y}^{\prime}=\left\{v \mid x u v \in \mathcal{P}_{x y}\right\}$.

Let $\{x, y\} \subset\{a, b, c\}$ and $z=\{a, b, c\} \backslash\{x, y\}$. Notice that each 2-path yuv $\in \mathcal{P}_{x y}$ $\left(x u v \in \mathcal{P}_{x y}\right)$, is contained in at most one good 3-path zyuv (respectively zxuv) whose first edge is in the triangle $a b c$. Indeed, since $u \in S_{x y}$, xyuv (respectively yxuv) is not a good 3 -path. Therefore, the number of good 3-paths whose first edge is in the triangle $a b c$, and whose third vertex is in $S_{x y}$ is at most $\left|\mathcal{P}_{x y}\right|$. The number of paths in $\mathcal{P}_{x y}$ that start with the vertex $x$ is less than $2\left|S_{x y}^{\prime}\right|+7680 \sqrt{n}$, by Lemma 19. Similarly, the number of paths in $\mathcal{P}_{x y}$ that start with the vertex $y$ is less than $2\left|S_{x y}^{\prime}\right|+7680 \sqrt{n}$. Since every path in $\mathcal{P}_{x y}$ starts with either $x$ or $y$, we have $\left|\mathcal{P}_{x y}\right|<4\left|S_{x y}^{\prime}\right|+15360 \sqrt{n}$. Therefore, for any $\{x, y\} \subset\{a, b, c\}$, the number of good 3-paths whose first edge is in the triangle $a b c$, and whose third vertex is in $S_{x y}$ is less than $4\left|S_{x y}^{\prime}\right|+15360 \sqrt{n}$.

In total, the number of good 3-paths whose first edge is in the triangle $a b c$ and whose third vertex is in $S_{a b} \cup S_{b c} \cup S_{a c}$ is at most

$$
4\left(\left|S_{a b}^{\prime}\right|+\left|S_{b c}^{\prime}\right|+\left|S_{a c}^{\prime}\right|\right)+46080 \sqrt{n} .
$$

Let $x \in\{a, b, c\}$ and $\{y, z\}=\{a, b, c\} \backslash\{x\}$. For any 2-path $x u v \in \mathcal{P}_{x}$ there are 2 good 3-paths with the first edge in the triangle $a b c$, namely yxuv and zxuv. So the total number of 3-paths whose first edge is in the triangle $a b c$ and whose third vertex is in $S_{a} \cup S_{b} \cup S_{c}$ is $2\left(\left|\mathcal{P}_{a}\right|+\left|\mathcal{P}_{b}\right|+\left|\mathcal{P}_{c}\right|\right)$, which is at most

$$
4\left(\left|S_{a}^{\prime}\right|+\left|S_{b}^{\prime}\right|+\left|S_{c}^{\prime}\right|\right)+46080 \sqrt{n}
$$

by Lemma 19.

Now we will prove that every vertex is in at most 2 of the sets $S_{a}^{\prime}, S_{b}^{\prime}, S_{c}^{\prime}, S_{a b}^{\prime}, S_{b c}^{\prime}, S_{a c}^{\prime}$. Let us assume by contradiction that a vertex $v \in V(G) \backslash\{a, b, c\}$ is in at least 3 of them. We claim that there do not exist 3 vertices $u_{a} \in N(a) \backslash\{b, c\}, u_{b} \in N(b) \backslash\{a, c\}$ and $u_{c} \in N(c) \backslash\{a, b\}$ such that $x u_{x} v$ is a good 3-path for each $x \in\{a, b, c\}$. Indeed, otherwise, consider hyperedges $h_{a}, h_{a}^{\prime}$ containing the pairs $a u_{a}$ and $u_{a} v$ respectively (since $a u_{a} v$ is a good 2-path, note that $h_{a} \neq h_{a}^{\prime}$ ), and hyperedges $h_{b}, h_{b}^{\prime}, h_{c}, h_{c}^{\prime}$ containing the pairs $b u_{b}, u_{b} v, c u_{c}, u_{c} v$ respectively. Then either $h_{a}^{\prime} \neq h_{b}^{\prime}$ or $h_{a}^{\prime} \neq h_{c}^{\prime}$, say $h_{a}^{\prime} \neq h_{b}^{\prime}$ without loss of generality. Then the hyperedges $h_{a}, h_{a}^{\prime}, h_{b}^{\prime}, h_{b}, a b c$ create a Berge 5 -cycle in $H$, a contradiction, proving that it is impossible to have 3 vertices $u_{a} \in N(a) \backslash\{b, c\}, u_{b} \in$ $N(b) \backslash\{a, c\}$ and $u_{c} \in N(c) \backslash\{a, b\}$ with the above mentioned property. Without loss of generality let us assume that there is no vertex $u_{a} \in N(a) \backslash\{b, c\}$ such that $a u_{a} v$ is a good 2-path - in other words, $v \notin S_{a}^{\prime} \cup S_{a b}^{\prime} \cup S_{a c}^{\prime}$. However, since we assumed that $v$ is contained in at least 3 of the sets $S_{a}^{\prime}, S_{b}^{\prime}, S_{c}^{\prime}, S_{a b}^{\prime}, S_{b c}^{\prime}, S_{a c}^{\prime}$, we can conclude that $v$ is contained in all 3 of the sets $S_{b}^{\prime}, S_{c}^{\prime}, S_{b c}^{\prime}$, i.e., there are vertices $u_{b} \in S_{b}, u_{c} \in S_{c}, u \in S_{b c}$ such that $v u_{b} b, v u_{c} c, v u b, v u c$ are good 2-paths. Using a similar argument as before, if $v u \in h, v u_{b} \in h_{b}$ and $v u_{c} \in h_{c}$, without loss of generality we can assume that $h \neq h_{b}$, so the hyperedges $a b c, h, h_{b}$ together with hyperedges containing $u c$ and $u_{b} b$ form a Berge 5 -cycle in $H$, a contradiction. 
So we proved that

$$
2\left|S_{a}^{\prime} \cup S_{b}^{\prime} \cup S_{c}^{\prime} \cup S_{a b}^{\prime} \cup S_{b c}^{\prime} \cup S_{a c}^{\prime}\right| \geqslant\left|S_{a}^{\prime}\right|+\left|S_{b}^{\prime}\right|+\left|S_{c}^{\prime}\right|+\left|S_{a b}^{\prime}\right|+\left|S_{b c}^{\prime}\right|+\left|S_{a c}^{\prime}\right|
$$

This together with (4) and (5), we get that the number of good 3-paths whose first edge is in the triangle $a b c$ is at most

$$
8\left|S_{a}^{\prime} \cup S_{b}^{\prime} \cup S_{c}^{\prime} \cup S_{a b}^{\prime} \cup S_{b c}^{\prime} \cup S_{a c}^{\prime}\right|+92160 \sqrt{n}<8 n+C_{1} \sqrt{n}
$$

for $C_{1}=92160$ and large enough $n$, finishing the proof of the claim.

Claim 23. Let $P=a b c$ be a 2-path and $P \in \mathcal{D}$. (By Observation 10 (b) abc $\in H$.) Then the number of good 3-paths whose first edge is ab or bc is at most $4 n+C_{2} \sqrt{n}$ for some constant $C_{2}>0$ and large enough $n$.

Proof. First we bound the number of 3-paths whose first edge is $a b$. Let $S_{a b}=N(a) \cap N(b)$. Let $S_{a}=N(a) \backslash(N(b) \cup\{b\})$ and $S_{b}=N(b) \backslash(N(a) \cup\{a\})$. For each $x \in\{a, b\}$, let $\mathcal{P}_{x}$ be the set of good 2-paths xuv where $u \in S_{x}$, and let $S_{x}^{\prime}=\left\{v \mid x u v \in \mathcal{P}_{x}\right\}$. The set of good 3-paths whose first edge is $a b$ is $\mathcal{P}_{a} \cup \mathcal{P}_{b}$, because the third vertex of a good 3-path starting with an edge $a b$ can not belong to $N(a) \cap N(b)$ by the definition of a good 3-path.

We claim that $\left|S_{a}^{\prime} \cap S_{b}^{\prime}\right| \leqslant 160 \sqrt{n}$. Let us assume by contradiction that $v_{0}, v_{1}, \ldots v_{k} \in$ $S_{a}^{\prime} \cap S_{b}^{\prime}$ for $k>160 \sqrt{n}$. For each vertex $v_{i}$ where $0 \leqslant i \leqslant k$, there are vertices $a_{i} \in S_{a}$ and $b_{i} \in S_{b}$ such that $a a_{i} v_{i}, b b_{i} v_{i}$ are good 2 -paths. For each $0 \leqslant i \leqslant k$, the hyperedge $a_{i} v_{i} b_{i}$ is in $H$, otherwise we can find distinct hyperedges containing the pairs $a a_{i}, a_{i} v_{i}, v_{i} b_{i}, b_{i} b$ and these hyperedges together with $a b c$, would form a Berge 5-cycle in $H$, a contradiction. We claim that there are $j, l \in\{0,1, \ldots, k\}$ such that $a_{j} \neq a_{l}$, otherwise there is a vertex $x$ such that $x=a_{i}$ for each $0 \leqslant i \leqslant k$. Then $x v_{i} \in G$ for each $0 \leqslant i \leqslant k$, so we get that $d_{G}(x)>k>160 \sqrt{n}$ which contradicts Claim 18.

So there are $j, l \in\{0,1, \ldots, k\}$ such that $a_{j} \neq a_{l}$ and $a_{j} v_{j} b_{j}, a_{l} v_{l} b_{l} \in H$. By observation 10 (b), there is a hyperedge $h \neq a b c$ such that $a c \subset h$. Clearly either $a_{j} \notin h$ or $a_{l} \notin h$. Without loss of generality let $a_{j} \notin h$, so there is a hyperedge $h_{a}$ with $a a_{j} \subset h_{a} \neq h$. Let $h_{b} \supset b_{j} b$, then the hyperedges $a b c, h, h_{a}, a_{j} v_{j} b_{j}, h_{b}$ form a Berge 5-cycle, a contradiction, proving that $\left|S_{a}^{\prime} \cap S_{b}^{\prime}\right| \leqslant 160 \sqrt{n}$.

Notice that $\left|S_{a}^{\prime}\right|+\left|S_{b}^{\prime}\right|=\left|S_{a}^{\prime} \cup S_{b}^{\prime}\right|+\left|S_{a}^{\prime} \cap S_{b}^{\prime}\right| \leqslant n+160 \sqrt{n}$. So by Lemma 19, we have $\left|\mathcal{P}_{a}\right|+\left|\mathcal{P}_{b}\right| \leqslant 2\left(\left|S_{a}^{\prime}\right|+\left|S_{b}^{\prime}\right|\right)+2 \cdot 7680 \sqrt{n} \leqslant 2(n+160 \sqrt{n})+2 \cdot 7680 \sqrt{n}=2 n+15680 \sqrt{n}$

for large enough $n$. So the number of good 3-paths whose first edge is $a b$ is at most $2 n+15680 \sqrt{n}$. By the same argument, the number of good 3-paths whose first edge is $b c$ is at most $2 n+15680 \sqrt{n}$. Their sum is at most $4 n+C_{2} \sqrt{n}$ for $C_{2}=31360$ and large enough $n$, as desired.

Claim 24. Let $\{a, b, c, d\}$ be the vertex set of a $K_{4}$ that belongs to $\mathcal{D}$. Let $F=K_{4}^{3}$ be a hypergraph on the vertex set $\{a, b, c, d\}$. (By Observation 10 (c) $F \subseteq H$.) Then the number of good 3-paths whose first edge belongs to $\partial F$ is at most $6 n+C_{3} \sqrt{n}$ for some constant $C_{3}>0$ and large enough $n$. 
Proof. First, let us observe that there is no Berge path of length 2,3 or 4 between distinct vertices $x, y \in\{a, b, c, d\}$ in the hypergraph $H \backslash F$, because otherwise this Berge path together with some edges of $F$ will form a Berge 5-cycle in $H$. This implies, that there is no path of length 3 or 4 between $x$ and $y$ in $G \backslash \partial F$, because otherwise we would find a Berge path of length 2,3 or 4 between $x$ and $y$ in $H \backslash F$.

Let $S=\{u \in V(H) \backslash\{a, b, c, d\} \mid \exists\{x, y\} \subset\{a, b, c, d\}, u \in N(x) \cap N(y)\}$. For each $x \in\{a, b, c, d\}$, let $S_{x}=N(x) \backslash(S \cup\{a, b, c, d\})$. Let $\mathcal{P}_{S}$ be the set of good 2-paths $x u v$ where $x \in\{a, b, c, d\}$ and $u \in S$. Let $S^{\prime}=\left\{v \mid x u v \in \mathcal{P}_{S}\right\}$. For each $x \in\{a, b, c, d\}$, let $\mathcal{P}_{x}$ be the set of good 2-paths $x u v$ where $u \in S_{x}$, and let $S_{x}^{\prime}=\left\{v \mid x u v \in \mathcal{P}_{x}\right\}$.

Let $v \in S^{\prime}$. By definition, there exists a pair of vertices $\{x, y\} \subset\{a, b, c, d\}$ and a vertex $u$, such that $x u v$ and yuv are good 2-paths.

Suppose that $z u^{\prime} v$ is a 2-path different from $x u v$ and yuv where $z \in\{a, b, c, d\}$. If $u^{\prime}=u$ then $z \notin\{x, y\}$ so there is a Berge 2-path between $x$ and $y$ or between $x$ and $z$ in $H \backslash F$, which is impossible. So $u \neq u^{\prime}$. Either $z \neq x$ or $z \neq y$, without loss of generality let us assume that $z \neq x$. Then $z u^{\prime} v u x$ is a path of length 4 in $G \backslash \partial F$, a contradiction. So for any $v \in S^{\prime}$ there are only 2 paths of $\mathcal{P}_{a} \cup \mathcal{P}_{b} \cup \mathcal{P}_{c} \cup \mathcal{P}_{d} \cup \mathcal{P}_{S}$ that contain $v$ as an end vertex - both of which are in $\mathcal{P}_{S}$ - which means that $v \notin S_{a}^{\prime} \cup S_{b}^{\prime} \cup S_{c}^{\prime} \cup S_{d}^{\prime}$, so $S^{\prime} \cap\left(S_{a}^{\prime} \cup S_{b}^{\prime} \cup S_{c}^{\prime} \cup S_{d}^{\prime}\right)=\emptyset$. Moreover,

$$
\left|\mathcal{P}_{S}\right| \leqslant 2\left|S^{\prime}\right|
$$

We claim that $S_{a}^{\prime}$ and $S_{b}^{\prime}$ are disjoint. Indeed, otherwise, if $v \in S_{a}^{\prime} \cap S_{b}^{\prime}$ there exists $x \in S_{a}$ and $y \in S_{b}$ such that $v x a$ and $v y b$ are paths in $G$, so there is a 4-path axvyb between vertices of $F$ in $G \backslash \partial F$, a contradiction. Similarly we can prove that $S_{a}^{\prime}, S_{b}^{\prime}, S_{c}^{\prime}$ and $S_{d}^{\prime}$ are pairwise disjoint. This shows that the sets $S^{\prime}, S_{a}^{\prime}, S_{b}^{\prime}, S_{c}^{\prime}$ and $S_{d}^{\prime}$ are pairwise disjoint. So we have

$$
\left|S^{\prime} \cup S_{a}^{\prime} \cup S_{b}^{\prime} \cup S_{c}^{\prime} \cup S_{d}^{\prime}\right|=\left|S^{\prime}\right|+\left|S_{a}^{\prime}\right|+\left|S_{b}^{\prime}\right|+\left|S_{c}^{\prime}\right|+\left|S_{d}^{\prime}\right|
$$

By Lemma 19, we have $\left|\mathcal{P}_{a}\right|+\left|\mathcal{P}_{b}\right|+\left|\mathcal{P}_{c}\right|+\left|\mathcal{P}_{d}\right| \leqslant 2\left(\left|S_{a}^{\prime}\right|+\left|S_{b}^{\prime}\right|+\left|S_{c}^{\prime}\right|+\left|S_{d}^{\prime}\right|\right)+4 \cdot 7680 \sqrt{n}$. Combining this inequality with (6), we get

$$
\left|\mathcal{P}_{S}\right|+\left|\mathcal{P}_{a}\right|+\left|\mathcal{P}_{b}\right|+\left|\mathcal{P}_{c}\right|+\left|\mathcal{P}_{d}\right| \leqslant 2\left|S^{\prime}\right|+2\left(\left|S_{a}^{\prime}\right|+\left|S_{b}^{\prime}\right|+\left|S_{c}^{\prime}\right|+\left|S_{d}^{\prime}\right|\right)+4 \cdot 7680 \sqrt{n}
$$

Combining (7) with (8) we get

$$
\left|\mathcal{P}_{S}\right|+\left|\mathcal{P}_{a}\right|+\left|\mathcal{P}_{b}\right|+\left|\mathcal{P}_{c}\right|+\left|\mathcal{P}_{d}\right| \leqslant 2\left|S^{\prime} \cup S_{a}^{\prime} \cup S_{b}^{\prime} \cup S_{c}^{\prime} \cup S_{d}^{\prime}\right|+30720 \sqrt{n}<2 n+30720 \sqrt{n},
$$

for large enough $n$.

Each 2-path in $\mathcal{P}_{S} \cup \mathcal{P}_{a} \cup \mathcal{P}_{b} \cup \mathcal{P}_{c} \cup \mathcal{P}_{d}$ can be extended to at most three good 3-paths whose first edge is in $\partial F$. (For example, auv $\in \mathcal{P}_{a}$ can be extended to bauv, cauv and dauv.) On the other hand, every good 3-path whose first edge is in $\partial F$ must contain a 2-path of $\mathcal{P}_{a} \cup \mathcal{P}_{b} \cup \mathcal{P}_{c} \cup \mathcal{P}_{d} \cup \mathcal{P}_{S}$ as a subpath. So the number of good 3-paths whose first edge is in $\partial F$ is at most $3\left|\mathcal{P}_{a} \cup \mathcal{P}_{b} \cup \mathcal{P}_{c} \cup \mathcal{P}_{d} \cup \mathcal{P}_{S}\right|=3\left(\left|\mathcal{P}_{S}\right|+\left|\mathcal{P}_{a}\right|+\left|\mathcal{P}_{b}\right|+\left|\mathcal{P}_{c}\right|+\left|\mathcal{P}_{d}\right|\right)$ which is at most $6 n+C_{3} \sqrt{n}$ by (9), for $C_{3}=92160$ and large enough $n$, proving the desired claim. 


\subsection{Combining bounds on the number of 3-paths}

Recall that $\alpha_{1}|G|, \alpha_{2}|G|,\left(1-\alpha_{1}-\alpha_{2}\right)|G|$ are the number of edges of $G$ that are contained in triangles, 2-paths and $K_{4}$ 's of the edge-decomposition $\mathcal{D}$ of $G$, respectively. Then the number of triangles, 2-paths and $K_{4}$ 's in $\mathcal{D}$ is $\alpha_{1}|G| / 3, \alpha_{2}|G| / 2$ and $\left(1-\alpha_{1}-\alpha_{2}\right)|G| / 6$ respectively. Therefore, using Claim 22, Claim 23 and Claim 24, the total number of (ordered) good 3-paths in $G$ is at most

$$
\begin{gathered}
\frac{\alpha_{1}}{3}|G|\left(8 n+C_{1} \sqrt{n}\right)+\frac{\alpha_{2}}{2}|G|\left(4 n+C_{2} \sqrt{n}\right)+\frac{\left(1-\alpha_{1}-\alpha_{2}\right)}{6}|G|\left(6 n+C_{3} \sqrt{n}\right) \leqslant \\
\leqslant|G| n\left(\frac{8 \alpha_{1}}{3}+2 \alpha_{2}+\left(1-\alpha_{1}-\alpha_{2}\right)\right)+\left(C_{1}+C_{2}+C_{3}\right) \sqrt{n}|G|= \\
=\frac{n^{2} \bar{d}_{G}}{2}\left(\frac{5 \alpha_{1}+3 \alpha_{2}+3}{3}\right)+\left(C_{1}+C_{2}+C_{3}\right) \frac{n^{3 / 2} \bar{d}_{G}}{2} .
\end{gathered}
$$

Combining this with the fact that the number of good 3-paths is at least $n \bar{d}_{G}^{3}-C_{0} n^{3 / 2} \bar{d}_{G}$ (see Claim 21), we get

$$
n \bar{d}_{G}^{3}-C_{0} n^{3 / 2} \bar{d}_{G} \leqslant \frac{n^{2} \bar{d}_{G}}{2}\left(\frac{5 \alpha_{1}+3 \alpha_{2}+3}{3}\right)+\left(C_{1}+C_{2}+C_{3}\right) \frac{n^{3 / 2} \bar{d}_{G}}{2} .
$$

Rearranging and dividing by $n \bar{d}_{G}$ on both sides, we get

$$
\bar{d}_{G}^{2} \leqslant\left(\frac{5 \alpha_{1}+3 \alpha_{2}+3}{6}\right) n+\frac{1}{2} \sqrt{n}\left(\left(C_{1}+C_{2}+C_{3}\right)+2 C_{0}\right) .
$$

Since $\left(5 \alpha_{1}+3 \alpha_{2}+3\right) / 6 \geqslant 1 / 2$, we may replace $1 / 2$ with $\left(5 \alpha_{1}+3 \alpha_{2}+3\right) / 6$ in the above inequality to obtain

$$
\bar{d}_{G}^{2} \leqslant\left(\frac{5 \alpha_{1}+3 \alpha_{2}+3}{6}\right) n\left(1+\frac{\left(C_{1}+C_{2}+C_{3}\right)+2 C_{0}}{\sqrt{n}}\right) .
$$

So letting $C_{4}=\left(C_{1}+C_{2}+C_{3}\right)+2 C_{0}$ we have,

$$
\bar{d}_{G} \leqslant \sqrt{1+\frac{C_{4}}{\sqrt{n}}} \sqrt{\frac{5 \alpha_{1}+3 \alpha_{2}+3}{6}} \sqrt{n}<\left(1+\frac{C_{4}}{2 \sqrt{n}}\right) \sqrt{\frac{5 \alpha_{1}+3 \alpha_{2}+3}{6}} \sqrt{n},
$$

for large enough $n$. By Claim 11, we have

$$
|H| \leqslant \frac{\alpha_{1}}{3}|G|+\frac{\alpha_{2}}{2}|G|+\frac{2\left(1-\alpha_{1}-\alpha_{2}\right)}{3}|G|=\frac{4-2 \alpha_{1}-\alpha_{2}}{6} \frac{n \bar{d}_{G}}{2} .
$$

Combining this with (10) we get

$$
|H| \leqslant\left(1+\frac{C_{4}}{2 \sqrt{n}}\right) \frac{\left(4-2 \alpha_{1}-\alpha_{2}\right)}{12} \sqrt{\frac{5 \alpha_{1}+3 \alpha_{2}+3}{6}} n^{3 / 2},
$$


for sufficiently large $n$. So we have

$$
\operatorname{ex}_{3}\left(n, C_{5}\right) \leqslant(1+o(1)) \frac{\left(4-2 \alpha_{1}-\alpha_{2}\right)}{12} \sqrt{\frac{5 \alpha_{1}+3 \alpha_{2}+3}{6}} n^{3 / 2} .
$$

The right hand side is maximized when $\alpha_{1}=0$ and $\alpha_{2}=2 / 3$, so we have

$$
\operatorname{ex}_{3}\left(n, C_{5}\right) \leqslant(1+o(1)) \frac{4-2 / 3}{12} \sqrt{\frac{5}{6}} n^{1.5}<(1+o(1)) 0.2536 n^{3 / 2}
$$

This finishes the proof.

\section{Acknowledgements}

We are grateful to the two anonymous referees for their valuable comments.

The research of the authors is partially supported by the National Research, Development and Innovation Office - NKFIH, grants K116769, SNN117879, KH126853. The research of the third author is also supported by IBS-R029-C1.

\section{References}

[1] B. Bollobás and E. Győri. "Pentagons vs. triangles." Discrete Mathematics, 308 (19) (2008), 4332-4336.

[2] R. G. Blakley and P. Roy. "A Hölder type inequality for symmetric matrices with nonnegative entries." Proceedings of the American Mathematical Society 16.6 (1965): $1244-1245$.

[3] B. Ergemlidze, E. Győri and A. Methuku. "Asymptotics for Turán numbers of cycles in 3-uniform linear hypergraphs." Journal of Combinatorial Theory, Series A 163 (2019), 163-181.

[4] B. Ergemlidze, E. Győri, A. Methuku, N. Salia, C. Tompkins and O. Zamora. "Avoiding long Berge cycles, the missing cases $k=r+1$ and $k=r+2$." Combinatorics, Probability and Computing (2018): 1-13.

[5] B. Ergemlidze, E. Győri, A. Methuku, N. Salia and C. Tompkins. "On 3-uniform hypergraphs avoiding a cycle of length four." Manuscript.

[6] B. Ergemlidze and A. Methuku "Triangles in $C_{5}$-free graphs and Hypergraphs of Girth Six." arXiv:1811.11873 (2018).

[7] Z. Füredi, A. Kostochka and R. Luo. "Avoiding long Berge cycles." arXiv: 1805.04195 (2018).

[8] Z. Füredi, A. Kostochka and R. Luo. "Avoiding long Berge cycles II, exact bounds for all n." arXiv:1807.06119 (2018).

[9] Z. Füredi and L. Özkahya. "On 3-uniform hypergraphs without a cycle of a given length." Discrete Applied Mathematics, 216 (2017), 582-588. 
[10] D. Gerbner, A. Methuku and C. Palmer. "General lemmas for Berge-Turán hypergraph problems." European Journal of Combinatorics 86 (2020): 103082.

[11] D. Gerbner, A. Methuku and M. Vizer. "Asymptotics for the Turán number of Berge$K_{2, t} . "$ Journal of Combinatorial Theory, Series B (2019).

[12] D. Gerbner and C. Palmer. "Extremal results for Berge-hypergraphs." SIAM Journal on Discrete Mathematics, 31.4 (2017), 2314-2327.

[13] D. Grósz, A. Methuku and C. Tompkins. "Uniformity thresholds for the asymptotic size of extremal Berge- $F$-free hypergraphs." European Journal of Combinatorics (2020): 103109 .

[14] A. Gyárfás. "The Turán number of Berge- $K_{4}$ in triple systems." SIAM Journal on Discrete Mathematics 33.1 (2019): 383-392.

[15] E. Győri. "Triangle-Free Hypergraphs." Combinatorics, Probability and Computing, 15 (1-2) (2006), 185-191.

[16] E. Győri and N. Lemons. "3-uniform hypergraphs avoiding a given odd cycle." Combinatorica 32.2 (2012), 187-203.

[17] E. Győri and N. Lemons. "Hypergraphs with no cycle of a given length." Combinatorics, Probability and Computing, 21 (2012), 193-201.

[18] E. Győri, N. Lemons, N. Salia and O. Zamora. "The Structure of Hypergraphs without long Berge cycles." arXiv:1812.10737 (2018).

[19] T. Jiang and J. Ma. "Cycles of given lengths in hypergraphs." Journal of Combinatorial Theory, Series B, 133 (2018): 54-77.

[20] A. Kostochka, and R. Luo. "On $r$-uniform hypergraphs with circumference less than r." arXiv: 1807.04683 (2018).

[21] F. Lazebnik and J. Verstraëte. "On hypergraphs of girth five." The Electronic Journal of Combinatorics 10 (2003): \#R25.

[22] C. Palmer, M. Tait, C. Timmons and A. Z. Wagner. "Turán numbers for Bergehypergraphs and related extremal problems." Discrete Mathematics 342.6 (2019): 1553-1563.

[23] I. Ruzsa and E. Szemerédi. "Triple systems with no six points carrying three triangles." in Combinatorics, Keszthely, Colloq. Math. Soc. J. Bolyai 18, Vol II (1976), 939-945.

[24] C. Timmons. "On $r$-uniform linear hypergraphs with no Berge- $K_{2, t}$. " The Electronic Journal of Combinatorics 24, no. 4 (2017): \#P4.34. 